\title{
Kernos
}

Revue internationale et pluridisciplinaire de religion grecque antique

12| 1999

Varia

\section{Chronique des rencontres scientifiques}

\section{André Motte}

\section{OpenEdition}

\section{Journals}

Édition électronique

URL : http://journals.openedition.org/kernos/726

DOI : $10.4000 /$ kernos.726

ISSN : 2034-7871

\section{Éditeur}

Centre international d'étude de la religion grecque antique

\section{Édition imprimée}

Date de publication : 1 janvier 1999

Pagination : 293-300

ISSN : 0776-3824

Référence électronique

André Motte, «Chronique des rencontres scientifiques », Kernos [En ligne], 12 | 1999, mis en ligne le 13 avril 2011, consulté le 16 septembre 2020. URL : http://journals.openedition.org/kernos/726 


\title{
Chronique des rencontres scientifiques
}

\author{
par André MotTe
}

Pour informer en temps utile les lecteurs de la revue, la rédaction soubaite être avertie aussitôt que possible des manifestations projetées; elle se fera un plaisir de les annoncer dans cette rubrique.

\section{A. Rencontres récentes (jusqu'au 31 mars 1999)}

Du 15 au 17 janvier 1998 s'est tenu, à l'Université de Naples, un colloque organisé par le Département des études du monde classique et de la Méditerranée antique sur le thème La letteratura pseudepigrafa nella cultura greca e romana. Parmi la quinzaine de communications présentées, on relevera ces trois titres qui ont rapport à la religion grecque :

F. Graf, Testo e rito. Il Corpus Eschatologicum degli Orfici; G. Ricciardelli, Gli Inmi orfici; Cl. Moreschini, $l l$ Corpus Hermeticum: perche Ermete Trismegisto?

De janvier à mars 1998 a eu lieu, à Faenza, un cycle de sept conférences organisé par les Amici dell'Arte sur le thème de La mitologia tra passato e futuro. Iside, Dioniso e Apollo :

E. Arslan, Iside; L. SAvelli, Viaggio a Delfi; G. Casadio, Dioniso e il vino e Dioniso tra ebbrezza ed entusiasmo; I. Chmassi, Miti, modelli e modi di Apollo, dio greco della conoscenza; P. Bernardini - E. Cingano, Apollo nella poesia greca arcaica; E. Bossi, Il mito di Apollo nellarte.

De janvier à mars 1998, la commune de Cecina a organisé, outre plusieurs visites guidées, un cycle de conférences sur le thème Dalla religione tradizionale etrusco-romana ai misteri :

G. CAtENi, La "Gens" etrusco-romana dei Caecina e le ume cinerarie di Volterra ad essa pertineti; F. FEDELI, La pittura etrusca arcaica e le sue correlazioni con l'ideologia funeraria; L. PALERMo, Le religioni misteriche nel mondo ellenistico e romano: $i$ misteri di Iside; G. Casado, Misteri dionisiaci: il vino di Dioniso.

Le 2 mai 1998 s'est tenue, à l'Université d'Édimbourg, une rencontre organisée par The Alison Johnson Memorial Conference sur le thème Religion and the Individual in Early Greece. Six communications ont été présentées :

R. HÄGG, Reflections on ancestors, beroes and the ordinary dead in Geometric and Arcbaic Greece; E. GEBHARD, Women's dedications at the Sanctuary of Poseidon at 
Istbmia; M. Dickie, Eumelus and the Bacchiad and Corinth in the 8th century B.C.; B. Kowalzig, Between oikos and polis: Individuals and Kinsbip Groups in Greek Cull; S. Humphreys, Kinship and dedications; T. Harrison, Belief and Ritual in Greek Religion.

Du 13 au 17 mai 1998 s'est tenu, à Louvain, un colloque international intitulé La théologie platonicienne et organisé par le Hoger Instituut voor Wijsbegeerte, De Wulf-Mansion-Center pour célébrer l'achèvement de la Theologia Platonica de Proclus, en l'honneur de ses éditeurs, H.D. Saffrey et L.G. Westerink $\uparrow$. Une trentaine de communications ont été présentées :

A. MotTe, Discours théologique et prière d'invocation. Proclus béritier et interprète de Platon; L. GERson, The Presence and the Absence of the Divine in the Platonic Tradition; J. Bussanich, Mystical Theology and Spiritual Experience in Proclus' Platonic Theology; D. O’Meara, La science métapbysique (tbéologie) de Proclus comme exercice spirituel; L. Siorvanes, The Platonic Theology: Truth or Fiction?; J. WhitTaker, Middle Platonic Antecedents; L. Brisson, Théologie platonicienne et Oracles Chaldaïques; H. BLumentHaL, Proclus and Plotinus, witb special reference to the Platonic Theology; A. SMITH, Porpbyry and the Platonic Theology; C. D'Ancona et C. LunA, Syrianus comme source textuelle et doctrinale de la Théologie platonicienne; S. Gersh, Proclus' Tbeological Methods; J. Cleary, Matbematical Methods in the Theology of Proclus; T. Kobusch, Das Eine ist nicht das Ganze. Die Idee der Totalität im Werk des Proklos, bes. in der Platonischen Theologie; F. Romano, L'idée de causalité cbez Proclus; D. Taonmina, Procédures de l'évidence dans la Théologie platonicienne; C. STEEL, Le Parménide, fondement de la Théologie platonicienne; J. Opsomer, Plato's Timaeus in the Platonic Theology; G. VAN Riel, Le Philèbe dans la Théologie platonicienne; A. Sheppard, Plato's Phaedrus in the Platonic Theology; J. Dillon, The Demilirge in the Platonic Theology; L. Couloubaritsis, Le statut du mythe chez Proclus; B. VAN DEN BERG, Touards the Paternel Harbour; on the Prayer in T.P. IV, 77, $20 \mathrm{ff}$. and Proclus' Sixth Hymn; Ph. HoffmanN, Remarques sur une triade chaldaique dans la Théologie platonicienne : Eros, Alètheia, Pistis; P. MeIJER, Some Unorthodox Views on the Nature of the One in Theologia Platonica II, 1 and 2; Y. Chitchaline, La doctrine des Hénades: Révélation divine? Découverte scientifique? Fiction consciente?; J. PÉpIN, La tbéologie symbolique des néoplatoniciens (Proclus, Denys); I. Perczel, Pseudo-Dionysius and the Platonic Theology; J. Combès, Damascius et la Théologie platonicienne; M. Cacouros, La réception de Proclus à Byzance aux XIIre et $X I V^{e} s$; W. Beierwaltes, Die Bedeutung der prokliscben Theologia Platonica für Nicolaus Cusanus; A. SEgond, Conclusion.

Les 16 et 17 juin 1998 s'est tenu, à l'Université de Salerne, un Seminario per il dottorato di Filologia classica organisé par le Dipartimento di Scienze dell'Antichità sur le thème Categorie del pensiero religioso e forme della letteratura nel mondo greco-latino. Outre les séminaires, cinq communications ont été présentées:

L. Troiani, Aspetti della religione nella letterature giudaico-ellenistica; V. Amoretxi, I Vangeli tra oralità e letteratura; J. ARONEN, Letteratura e religione a Roma repubblicana; M.G. LANCELlotTi, Letteratura e realtà nei papiri magici dell' Egitto romano; G. Lieberg, L'indole degli dei nelle storie d'amore delle Metamorfosi ovidiane.

Du 22 au 23 août 1998 s'est tenu, à Ascona-Ticino, la Session d'Eranos sur le thème Le langage des masques. Les communications suivantes ont été présentées: 
E. Srmon, Stumme Masken und sprecbende Gesicbter. Die Arcbäologie griecbiscber und römiscber Masken; R. OHASHI, Die Welt als Maske. Japonischer Beitrag zum Problem der Spracbe; R. BodeI, La Maschara sulla Came. Per una Topografia del Volto; K RöTTGER, Zwiscbenspiele in Zwiscbenträumen. Das Sprecben mit Masken im zeitgenössiscben Theater Lateinamerikas; D. Carasco, Masking Deatb, Masking Life. Days of the Death in. Mexico; A. Juranville, Masque, sexe et bistoire. La vérité du masque à l'époque de la psychoanalyse; M. Barasch, Animal Masks in European Imagery; J. Assmann, Du Siebst Mit Dem Kopf Eines Gottes. Gesicht und Maske im alt-ägyptischen Kult.

Du 30 septembre au 2 octobre 1998 s'est tenu, à Padoue, un Symposium international organisé par l'Istituto Sperimentale per la Viticoltura, à l'occasion de son $75 \mathrm{e}$ anniversaire, et par le Dipartimento di Scienze dell'Antichità de l'Université de Padoue sur le thème L'avventura del vino nel bacino del mediterraneo. Itinerari storici ed archeologici prima e dopo Roma.

Le 21 octobre 1998 s'est tenue, à l'Université de Liège, la $13^{e}$ rencontre organisée par le Groupe de contact interuniversitaire pour l'étude de la religion grecque (F.N.R.S.). Irad MALKIN, professeur à l'Université de Tel-Aviv, y a fait une conférence intitulée Les retours d'Ulysse. Colonisation et etbnicité.

Le 24 octobre 1998 s'est tenu, au Collège de France, un colloque organisé par la Société Ernest Renan sur le thème Mystères et initiation. Neuf communications ont été présentées:

C. Traunecker, L'initiation en Égypte ancienne; L. BruIT-ZaIdman, Mystères et initiation en Grèce ancienne; F. Joun, Mystères et rites oraculaires : l'oracle des morts à Épbyra d'Épire; G. SAUron, La grande fresque de la villa des mystères : mémoires d'une dévote de Dionysos; E.-M. LAPERrousaz, L'initiation selon les manuscrits de la mer Morte. La communauté essénienne du "Mâtre de justice de justice" : communauté initiatique des "Fils de Lumière"; I. Melıoff, Un Islam en marge de l'Tslam : l'Alévisme; A.-M. WoHrer, Rituels d'initiation pour intégrer les ordres de cbevalerie chez les Mexicains; G. Dieterlen, Initiation et système de pensée en Afrique occidentale; Ch. Porcet, La Franc-maçonnerie.

Du 4 au 6 novembre 1998 s'est tenu, à l'Université Libre de Bruxelles, sous l'égide de la Société Belgo-Luxembourgeoise d'histoire des religions, un colloque international sur le thème Les démons dans les mystères. Dix-huit communications ont été présentées:

J. Ries, Introduction au thème du colloque; R. Turcan, Les démons et la crise du paganisme gréco-romain; P. JUDET DE LA COMBE, Un débat implicite sur les démons : Escbyle, Orestie, et Empédocle, Catharmes; Ph. Hoffmann, La présence du démon de Socrate dans la philosophie antique; B. Decharneux, Jésus, chasseur de démons. Témoignages de la littérature chrétienne des origines; J. Opsomer, La démonologie dans le stolicisme; Ch. M. TERnEs, Êtres intermédiaires sur le cénotaphe d'Igel. Influences 'orientales' en Gaule Belgique; C. Deroux, Les démons de la nuit : du paganisme au christianisme; L. Rızzerio, Rôle des êtres intermédiaires dans les cbemins de la connaissance chez Clément d'Alexandrie; J. Boulogne, Les fonctions de la démonologie chez Plutar- 
que; Th. Thomas, La chute d'après Origène; A. Petrt, Démonologie néopythagoricienne; L. Couloubaritsis, Fondements de la démonologie chez Plotin; M. Broze et C. Van Liefferinge, Er le Pampbilien : de l'âme angélique chez Jamblique et Prochus; F. Labrique, Chamanisme ou théurgie?; A. LERNOULD, Le traitement philosophique de la question des démons dans le néoplatonisme tardif; $\mathrm{M}$. Tardeu, Les bypothèses télestiques de l'empereur Julien; A. Motre, Conclusions.

Le 18 novembre 1998 s'est tenue, à l'Université de Liège, une journée d'étude organisée par le Groupe de contact F.N.R.S d'Études chypriotes sur le thème Les sanctuaires de Chypre. A.-M. Collombier, professeur à l'Université de Nancy, y a fait une conférence sur Les sanctuaires de Cbypre aux périodes archaïque et classique et Cl. BauraIn, professeur à l'Université de Liège, a fait une conférence sur le thème Sanctuaires et religion à Chypre à l'âge du Bronze.

Du 9 au 11 décembre 1998 s'est tenu, à l'Université Blaise Pascal de Clermont-Ferrand, un colloque international organisé en l'honneur de Christoph W. Clairmont par le Centre de Recherches sur les Civilisations Antiques sur le thème Les pierres de l'offrande. Une trentaine de communications y ont été présentées :

G. Hoffmann, Présentation de l'oeuure de Christoph W. Clairmont; Ch. W. Clairmont, L'étude des reliefs funéraires grecs : une rétrospective (1951-1997); J. BERGEMANN, Die Datenbank der attischen Grabreliefs: neue Möglichkeiten für die ikonographische und sozialgeschichtliche Forschung; B. HoLtzmanN, Les stèles funéraires de Thasos, du vie au $I^{e}$ siècle : traditions insulaires et types attiques; D. Auserve-Berranger, Les stèles funéraires de linle de Paros entre 650 et 450; N. Demand, The cultural Construction of the Body and Representational Art: Greek Physicians and Sculptors; B. SCHMALTZ, Re-using Classical Gravereliefs; R. Posamentir, Painted Attic Gravestones of the Classical Period and their Position within Classical Funerary Art; K. STEArs Some monuments are More Equal than Others: Reflections on Fourth Century Athenian Funerary Reliefs; V. DaSEN, Les jumeaux dans l'imaginaire funéraire antique; T. Lochman, Les stèles funéraires au Musée des Antiquités de Bâle; J.-M. Rouquette, Présentation du Musée de l'Arles antique; St. Georgoud, Le culte des ancêtres: le cas des Tritopatores de Sélinonte; M. Daumas, Stèles funéraires d'initiés des Cabires; A. Helmis, Le droil des sépultures en Grèce ancienne; M. Moltesen, Greek or Roman, A Group of Perbaps Neo-attic Reliefs found in Rome; O. Cavalier, A propos d'une stèle récemment acquise par le Musée Calvet; M.-Th. Le Dinahet, L'image des fillettes dans les inscriptions et reliefs funéraires déliens (époque bellénistique); A. Verbanck-Pí́rard, Stèles en salles. Comment te dire adieu?; S. VILATTE, Une stèle imaginaire : les dialogues platoniciens en l'bonneur de Socrate; Chr. Vendries, Une lyre sur la stèle de M. Cincios Nigrinos, soldat des cohortes urbaines; O. Rounult, Pratique funéraire et culte des ancêtres dans le Moyen-Euphrate antique; G. Galliano et V. Gay, Les stèles funéraires égyptiennes du Musée des Beaux-Arts de Lyon; A. SArtre, La stèle funéraire en Syrie à l'époque romaine; J. Delclos, Les monuments funéraires de Vétérans à Sens sous le Haut-Empire; L. LAmoINe, Iconographie funéraire et bonneurs municipaux en Narbonnaise; Ch. GuITTARD, Les Elogia Scipionum; $\mathrm{S}$. Labarre, Vie terrestre et vie céleste dans les épitaphes mérovingiennes de Venance Fortunat (Vte siècle); P. Garrigues, Un vol de libellule au fond d'un soir d'été : poétique de l'épigramme; A. FIASCHI, Des stèles de sons élevées à la mémore des maitres disparus : les tombeaux dans la musique française du XVITe et de la première moitié du XVIII siècles; Th. Dutort, Monuments funéraires et épitaphes dans l'Angleterre de la fin du XVII siècle; V. Deshoulieres, Images d'anges : du bilan à l'adieu. Propositions pour une poésie stélaire; V. Partensky, Stèles : le repos et l'exode. Die Juden von Zirndorf de Jacob 
Wassemann; A. VerLet, Que c'est triste un jardin sans tombeaux ! : les fleurs de ruine de Cbateaubriand à Colette; B. Westrhal, Les stèles d'Ovide dans le dernier des mondes de Ransmayr; O. CHAZAUd, La généalogie de la morale : du corps et du tombeau; S. ForeroMendozA, Sub specie mortis : les rituels funèbres de Boltanski.

Du 24 au 28 février 1999, la Provincia Regionale di Agrigento a organisé dans cette ville un important congrès international sur le thème $\boldsymbol{L} \boldsymbol{a}$ Sicilia dei due Dionisî. Plus de quarante communications y ont été présentées sur des sujets variés relevant de la philosophie, de la mythologie, de l'économie, de l'histoire politique, de l'archéologie et de l'histoire de l'art.

Du 25 au 27 mars 1999 s'est tenu, à l'Université Paul-Valéry de Montpellier, un colloque international organisé par le Séminaire d'Étude des Mentalités Antiques sur le thème de La magie. Une quarantaine de communications y ont été présentées:

J. Bотт́́ro, Magie, exorcisme et religion en Mésopotamie; S. Aufrère, Le dernier Nectanébo et la tradition bellénistique de la magie égyptienne; N. Bosson, A la croisée des chemins. Fondements de la magie copte traditionnelle (VIte-VIII siècles); P. BrianT, Les mages à l'époque achéménide; J. BREMMER, La confrontation entre l'apôtre Pierre et Simon le Magicien; B. SeRGENT, Maponos : la malédiction; F. GRAF, Une bistoire magique. Les phases constitutives de l'bistoire de la magie antique; P. Wathelet, Dieux et enchantements dans l'épopée boméirque; G. Karsai, La magie dans l'Odyssée : Cicrcé; F. Joun, Pratiques de magie et drame satyrique; A. Morte, A propos de la magie chez Platon: le sophiste-sorcier et le philosophe-enchanteur; B. PÉREZ, La magie d'Éros (Platon, Banquet); J.-M. Renaud, Magie et imprécations dans les mythes grecs: le cas d'Althaia; P. Sauzeau, Hékatè; D. Briquel, Haruspices et magie; l'évolution de la discipline étrusque dans l'Antiquité tardive; G. CAPDEville, La magie de Manto d'après l'Edipe de Sénèque; D. Pralon, Les Magiciennes de Théocrite; A. Moreau, Médée la magicienne au prometheion, un monde de l'entre-deux (Apollonios de Rhodes, Argonautiques, III, 828-870); $\mathrm{R}$. Buxton, Les yeux de Médée : psycbologie et magie dans les Argonautiques d'Apollonios de Rhode; A. BERNAND, La voix des exchis; S.I. Johnston, Les sacrifices dans les papyrus magiques grecs; F. Frazier, L'ame ensorcelée : magie des Muses et magie de l'amour selon Plutarque; J. Boulogne, Plutarque et les Mages; A. Zucker, Magie instinctive et artificielle chez Elien; M.-L.. Freyburger, Dion Cassius et la magie; C. Van LigfFeringe, Magie et théurgie chez Jamblique; B. SCHouler, Intervention divine et pratique magique. Le point de vue d'Eunape et de Libanios; H. Frangoulrs, Dionysos dans les Dionysiaques de Nonnos de Panopolis : dieu ou sorcier?; M.G. LANCELLotTI, Problèmes métbodologiques dans la constitution d'un corpus des gemmes magiques; $\mathrm{R}$. BOYER, Savoir et pouvoir : la magie dans l'Edda poétique; G. FreYBurger, Prière et magie à Rome : le carmen et l'oraison silencieuse; A. Novara, Magie, amour et bumour chez Properce; Ch. Segal, Tantum medicamenta possunt: Magic in Ovid's Metamorphoses; B. Bakhouche, Les noces de Philologie et de Mercure, II, 141ss.; E. Diouf, Magie et droit chez Pline l'Ancien; N. MÉTHx, Magie, religion et philosophie au second siècle de notre ère. A propos du Dieu-Roi d'Apulée; F. FAuQuier, La magie dans le Picatrix; L. BaldiniMoscadi, Les métamorphoses de la magie : la Médée-virgo de Valerius Flaccus; F. Dubost, La magicienne amoureuse dans le récit médiéval (XII ${ }^{e}-X I I^{e}$ siècles); J.P. BoudeT, L'ars notoria au Moyen Âge : une résurgence de la théurgie antique?; D. Vazeilles, Du cbamanisme au néo-chamanisme et au Nouvel Age: savoir, magie, religion ou quête spirituelle?; J. BONNET, Les femmes rurales et leur magie; M. MAC Donald, la représentation de Médée la magicienne dans l'opéra; M. Schneinfeigel, Cinéma et magie. Des Actes sont prévus. 
Durant l'année académique 1998-1999 s'est tenu, à l'Université de Paris IV-Sorbonne, un cycle de six conférences d'initiation organisé par les Écoles doctorales «Les mondes de l'antiquité » et « Histoire des religions " sur le thème Les religions de l'Europe ancienne :

G. CAPDEville, La religion minoenne et La religion étrusque; J.-J. MAFFre, La religion grecque classique; D. PORTE, La religion romaine; D. BRIQUEL, Les religions italiques; B. SERGENT, La religion celtique.

\section{B. Rencontres annoncées (à partir du $1^{\mathrm{er}}$ avril 1999)}

Du 21 au 23 mai 1999 tiendra, à Rome, un colloque international organisé par l'Istituto per la civilà fenica et l'Istituto per gli studi micenei ed egeo-anatolici du Consiglio Nazionale delle Ricerche sur le thème La questione delle influenze vicino-orientali sulla religione greca. Stato degli studi e prospettive della ricerca. Organisation : S. RIBICHINI, M. Rocchr et P. XELLA, Istituto per la Civiltà fenica e punica, CNR, Area della Ricerca di Roma, Via Salaria, C.P. 10, I - 00016 Monterotondo Stazione (Roma).

Walter BURKERT (Zürich), La religione greca all'ombra dell'Oriente: i livelli dei contatti e degli influssi; Lambert IseBAERT (Namur), Fr. Max Müller et la religion grecque; Nota Kourou (Athens), Gods, Heroes, and Trees in Greek Art: Mycenaean Versus Oriental Traditions; Cecilia BeER (Stockholm), Le rôle de Cbypre dans la transmission de pratiques cultuelles proche-orientales en Grèce (Âge du Fer); Maria Rosaria Belgiorno (Roma), C'è un nesso tra Ammone e il vello d'oro?; Franca Daddi Pecchioli (Firenze) - Anna Maria Polvani (Firenze), Temi di mitologia anatolica tra Oriente e Occidente - I. Il dio scomparso; II - Le teomachie; Mauro Giorgreri (Roma), Aspetti magico-religiosi del giuramento presso gli Ittiti e $i$ Greci; Sergio Ribichini (Roma), "Fascino" dall'Oriente e prime lezioni di magia; Philippe Borgeaud (Genève), La Mère des dieux entre le Proche-Orient et la Grèce; Maria Rocchl (Roma), Origini e ruoli dei monti nei miti greci e vicino-orientali; Domenico Musti (Roma), Aspetti del culto cabirico; Manuela MARI (Roma), Gli studi sul santuario e $i$ culti di Samotracia: prospettive e problemi; Vinciane Pirenne (Liège), La Crète et les îles de l'Egée dans la genèse d'Aphrodite; Pietro Vannicelli (Roma), Eracle e la cronologia orientale nella storiografia greca di $V$ secolo a.C.; Corinne BonNET (Roma/ Cosenza) - Colette Jourdain-ANNequin (Grenoble), Images et fonctions d'Héraclès: les modèles orientaux et leur interprétation; Patrick MarCHETTI (Namur), Éléments orientaux dans la religion argienne; Marie-Françoise BAstez. (Paris), La vie associative dans les sanctuaires orientaux du monde grec, traditions orientales et modèles grecs; Anna Maria Gloria Capomacchia (Roma), Motivi orientali nel teatro tragico greco; Ileana CHIRAssi Colombo (Macerata), L'Oriente di Medea: un'icona greca da Euripide a Pasolini; Carlo Brillante (Venezia), Eroi orientali nelle genealogie greche; Paolo Merlo (Roma) - Paolo Xella (Roma/Tübingen), D'Ervin Robde aux Rapiuma ougaritiques. Antécédents orientaux des béros grecs?; Amalia Margherita Cirio Medaglia (Roma), Nuovi dati sul culto degli eroi: un'interpretazione di Alceo $140 \mathrm{~V}$.; Wolfgang RöLlig (Tübingen), Myths about the Netberworld in the Ancient Near East and their Counterparts in the Greek Religion; Maria Cruz Marin Ceballos (Sevilla), Fenicios y Griegos en el ámbito ibérico: contactos religiosos; Gabriella Scandone Matthiae (Roma), Osservazioni egittologiche ad Erodoto; Cristiano Grottanelli (Pisa), Rapporti tra umanità e dèi nelle iscrizioni di dono semitiche, greche e latine; Vassilis Aravantinos (Thebes), Oriental Elements in the Mycenaean Findings at Thebes; Enrico SCAFA (Roma) - Maria Luisa Alfe' (Roma), Analogie nelle organizzazioni templari orientali e micenee; Paola Negri Scafa (Roma), Aspetti del sacerdozio femminile nel Vicino Oriente antico e nel mondo miceneo; Silvia Maria CHIODI (Roma) - Francesco Adorno (Firenze) - Giovanni Pettinato (Roma), Lo studio del cielo tra scienza e religione ovvero la trasmissione delle credenze mesopotamiche nel mondo 
greco; Antonio Panaino (Ravenna), Riflessioni sul concetto di "Anno Cosmico"; Paolo Xella (Roma/Tübingen), Conclusioni del Colloquio.

Du 24 au 26 mai 1999 se tiendra, à l'Université de Valladolid, le VII colloque organisé par le Centre International d'Étude de la Religion Grecque Antique sur le thème Héros et béroïnes dans les mythes et les cultes grecs. Organisation: E. Suárez de la Torre, Departamento de Filología Griega, Facultad de Filosofía y Letras, Universidad de Valladolid, E 47002 Valladolid, Tél. : + 34983423129 / Fax : + 34983423007 / E-mail : esuarez@vasertel.es.

F. BADER (Paris), Le béros Mérion; C. BARRigón (Valladolid), La designación de béroes y beroínas en la lírica griega; A. Bernardini (Urbino), La donna e l'eroe nel mito di Eracle; A. Blomart (Harvard), Les manières grecques de déplacer des béros: modalités religieuses et motivations politiques; J.L. Calvo MarTínez (Granada), La katábasis beroica; L. Couloubaritsis (Bruxelles), La transfiguration du béros en pbilosophe; B. Decharneux (Bruxelles), Moise, un béros bellénisé insolite?; M.-P. De Hoz García-Bellido (Salamanca), El culto a los béroes en Asia Menor según Estrabón; R. Di Donato (Pisa), I culti degli eroi ad Atene: evidenza e modelli; F. Díaz Platas (Santiago de Compostela), Ninfas y beroinas en la épica griega arcaica; F. Diez DE VELAsco (La Laguna), Iconografía de Heracles en el jardin de las Hespérides; G. Еквотн (Paris), The Role of Blood Libations in Greek Hero-Cults; M. Garcia Teijeiro - M. T. Molinos Tejada (Valladolid), Los béroes malvados; ZL. Gocevi (Sophie), Le héros dans la religion grecque et thrace; F. GraF (Bâle), Transformations merveilleuses: les béros nouveaux de l'époque bellénistique; R. HäGG (Göteborg), Early Hero Cults: The Archaeological Evidence; G. Hoffmann (Paris), Le fait d'armes comme source de l'béroisation dans la cité classique; M.-C. Herrero Ingelmo (Valladolid), Los béroes epónimos; A. Iriarte (País Vasco), Ismene, Crisótemis y sus bermanas; F. Jovan (Paris), Héros tragiques et deus ex machina dans quelques tragédies d'Euripide; Y. LAFOND (Arras), Figures béroïques dans la religion civique en Grèce à l'époque impériale romaine; L.-M. L'Homme-Wéry (Liège), Les béros de Salamine en Attique. Culte et intégration politique; J. A. López Férez (Madrid), Aquiles en Eurípides; S. Montero Herrero (Madrid), Los manteis griegos, héroes de guerra; A. Morenu (Montpellier), Actéon: des avatars du mythe aux variations littéraires; A. Moтte (Liège), La catégorie de l'béroique chez Platon; E. Moutsopoulos (Athènes), L'univers musical des béroines d'Eschyle; A. Pérez Jiménez (Málaga), Perfiles bumanos de un béroe; Plutarco y su imagen de Teseo; M. Pí́rart (Fribourg), héros fondateurs, béros civilisateurs. La rivalité entre Argos et Atbènes vue par Pausanias; E.-A. Ramos Jurado (Cádiz), La integración de la clase beroica en el pensamiento griego de la Antigïedad tardia; I. RATINAUD (Grenoble), Héros bomériques et cultes d'époque géométrique; M. Rocchi (Roma), Gli eroi e le origini delle montagne; I. Rodríguez Moreno (Cádiz), El béroe como metaxy entre el bombre y la divinidad en el pensamiento griego; A. Serghidou (Rethymnon), Dégradation du béros et politiques de l'exclusion dans la tragédie grecque; I. SHopova (Sofia), Heracles and Dionysos in Roman Thrace: interpretatio Graeca vel Thracica?; P. Somville (Liège), Héro et Léandre: un exemple d'béroísation tardive; I. TAssignoN (Bruxelles), Les béros fondateurs face à Dionysos; N. Theodossiev (Sofia), Monumental Tombs and Hero Cults in Thrace During the 5th-3rd Centuries B.C.; A. VerBaNCK-PIÉRARD (Musée de Mariemont), Les béros guérisseurs: des dieux comme les autres !; E. Voutiras (Thessalonique), Le cadaure et le serpent, ou l'béroisation manquée de Cléomène de Sparte; P. Wathelet (Liège), La double initiation d'Acbille dans l'lliade; B. ZimmermanN (Freiburg), Heroen im Ditbyrambos.

Du 24 au 26 octobre 1999 se tiendra, à Luxembourg, un colloque organisé par l'Association Homo religiosus, Luxembourg sur le thème Dieux, 
fêtes, sacré dans la Grèce et la Rome antiques. Organisation : Prof. Ch. M. Ternes, Centre universitaire de Luxembourg, av. de la Fayencerie, $162 \mathrm{~A}$, L-1511 Luxembourg. Fax : +352 466644 213; E-Mail : cmt@csi.com.

R. Turcan, La fête dans les rituels initiatiques; G. CaPdeville, L'épiphanie du dieu dans l'arbre et le culte de l'arbre sacré en Crète et à Chypre; D. Aubriot, Autour de quelques fêtes épiques et lyriques. Homère et Pindare; I. TASsignon, Les katagogies dionysiaques en Asie Mineure; F. Dunand, Fêtes et réveil religieux dans les cités grecques à l'époque bellénistique; V. Pirenne-Delforge, La fête dans. la Périégèse de Pausanias; A. MotTE, Significations de la fête grecque; D. Briquel, Vertumnus / Vertumna. L'origine des fêtes fédérales au Latium; Y. LEHMANN, Les livres sibyllins; Ch.M. TERNEs, Le festif en milieu gallo-romain : arcbéologie, épigraphie, iconographie; Th.P. OsBorNe, Le conflit des cultes dns l'Apocalypse de Jean; J. RiEs, Sacralisation dans le culte impérial; Ch. GuITTARD, La prière dans les cérémonies des Jeux Séculaires augustéens; M. GschaId, Die Götterverebrung in des Provinzen Raetien, Noricum, Pannonien und ibr festlicher Aspekt.

Le $\mathrm{VI}^{\mathrm{e}}$ Symposium platonicum organisé par la Société platonicienne internationale aura lieu à Jérusalem, en août 2001, et sera consacré aux Lois de Platon. Précisions dans le prochain numéro. 